\title{
Numerical solution of time fractional Burgers equation
}

\author{
A. Esen \\ Department of Mathematics, \\ Faculty of Science and Art, \\ Inönü University, Turkey
}

\author{
O. Tasbozan \\ Department of Mathematics, \\ Faculty of Science and Art, \\ Mustafa Kemal University, Turkey \\ email: otasbozan@mku.edu.tr
}

\begin{abstract}
In this article, the time fractional order Burgers equation has been solved by quadratic B-spline Galerkin method. This method has been applied to three model problems. The obtained numerical solutions and error norms $L_{2}$ and $L_{\infty}$ have been presented in tables. Absolute error graphics as well as those of exact and numerical solutions have been given.
\end{abstract}

\section{Introduction}

The Burgers equation is a nonlinear equation for diffusive waves in fluid dynamics. It exists various physical problems such as one-dimensional sound waves in a viscous medium, waves in fluid filled viscous elastic tubes, shock waves in a viscous medium and magnetohy-drodynamic waves in a medium with finite electrical conductivity, turbulence etc. [1]. Numerical solutions of the Burgers equation in the literature have been obtained using different methods and techniques $[2,3,4,5,6,7]$. In addition, the fractional order Burgers equation has been solved by many authors $[8,9,10,11,12,13,14]$.

The main idea underlying the finite element method, finite element nodes that are related to entire of the equivalent system can discretize the problem

2010 Mathematics Subject Classification: 97N40, 65N30, 65D07,74S05

Key words and phrases: finite element method, Galerkin method, time fractional Burgers equation, quadratic B-spline 
area and the most appropriate one will be a true physical behavioral model to choose the most appropriate type of element. Thus with the help of this method, an equation which is hard to solve can be turned into a few solvable set of equations. Finite element adjustable yet small enough and large enough to reduce computation load of the problem in available sizes[15].

Due to its capacity for non-integer order derivatives and integrals of fractional calculus have become an indispensable part of applied mathematics. Applications of differentiation and integration with non-integer orders can be traced back to premature in history, so it can be said that it is not new[16]. Many different techniques and methods of dealing with fractional differential equations resulting analytical and numerical solutions can be found in a wide variety of studies in the literature $[17,18,19,20,21,22,23,24,25,26,27,28$, $29,30,31]$.

In this paper, we consider the time fractional Burgers equation for $0<\gamma<1$

$$
\frac{\partial^{\gamma} \mathrm{u}(x, t)}{\partial t^{\gamma}}+\mathrm{U}(x, t) \frac{\partial \mathrm{U}(x, t)}{\partial x}-v \frac{\partial^{2} \mathrm{u}(x, t)}{\partial x^{2}}=f(x, t)
$$

with the boundary conditions

$$
\mathrm{u}(\mathrm{a}, \mathrm{t})=\mathrm{h}_{1}(\mathrm{t}), \quad \mathrm{u}(\mathrm{b}, \mathrm{t})=\mathrm{h}_{2}(\mathrm{t}), \quad \mathrm{t} \geq 0
$$

and the initial condition

$$
\mathrm{u}(x, 0)=\mathrm{g}(\mathrm{x}), \quad \mathrm{a} \leq \mathrm{x} \leq \mathrm{b},
$$

where $v$ is a viscosity parameter and

$$
\frac{\partial^{\gamma} \mathrm{U}(x, t)}{\partial t^{\gamma}}=\frac{1}{\Gamma(1-\gamma)} \int_{0}^{t}(t-\tau)^{-\gamma} \frac{\partial \mathrm{U}(x, \tau)}{\partial \tau} \mathrm{d} \tau
$$

is the Caputo fractional derivative [32]. In this paper, to achieve a finite element layout of the time fractional Burgers equation, Caputo fractional derivative formulation can be discretizated through L1 formulae [17]:

$$
\left.\frac{\partial^{\gamma} f(t)}{\partial t^{\gamma}}\right|_{t_{m}}=\frac{(\Delta t)^{-\gamma}}{\Gamma(2-\gamma)} \sum_{k=0}^{m-1}\left[(k+1)^{1-\gamma}-k^{1-\gamma}\right]\left[f\left(t_{m-k}\right)-f\left(t_{m-1-k}\right)\right] .
$$

\section{Quadratic B-spline finite element Galerkin solu- tions}

In this section, the time fractional Burgers equation has been solved by quadratic B-spline Galerkin method. For this firstly, Eq. (1) is multiplied with weigh 
function $W(x)$ and then integrated over the region, we get

$$
\int_{a}^{b}\left(\frac{\partial^{\gamma} u}{\partial t^{\gamma}}+u \frac{\partial u}{\partial x}-v \frac{\partial^{2} u}{\partial x^{2}}\right) w d x=\int_{a}^{b} w f(x, t) d x .
$$

In Eq. (4), if we apply partial integration, we have weak form

$$
\int_{x_{m}}^{x_{m+1}}\left(W \frac{\partial^{\gamma} u}{\partial t^{\gamma}}+W u \frac{\partial u}{\partial x}+v \frac{\partial W}{\partial x} \frac{\partial u}{\partial x}\right) d x=\left.v W \frac{\partial u}{\partial x}\right|_{x_{m}} ^{x_{m+1}}+\int_{x_{m}}^{x_{m+1}} W f(x, t) d x
$$

which is on only one of the $\left[x_{m}, x_{m+1}\right]$ finite element of Eq. (1). To modify the global coordinate system to the local one we did made use of transformation $\xi=x-x_{m}$. So, Eq. (5) turns into the form

$$
\int_{0}^{h}\left(W \frac{\partial^{\gamma} u}{\partial t^{\gamma}}+W u \frac{\partial u}{\partial \xi}+v \frac{\partial W}{\partial \xi} \frac{\partial u}{\partial \xi}\right) d \xi=\left.v W \frac{\partial u}{\partial \xi}\right|_{0} ^{h}+\int_{0}^{h} W \tilde{f}(\xi, t) d \xi .
$$

We describe quadratic B-spline base functions. Let us consider the interval $[a, b]$ is partitioned into $\mathrm{N}$ finite elements of uniformly equal length by the knots $x_{m}, m=0,1,2, \ldots, N$ such that $a=x_{0}<x_{1} \cdots<x_{N}=b$ and $h=$ $x_{m+1}-x_{m}$. The quadratic B-splines $Q_{m}(x),(m=-1(1) N)$, at the knots $x_{m}$ are defined over the interval $[a, b]$ by [33]

$$
Q_{m}(x)=\frac{1}{h^{2}}\left\{\begin{array}{lc}
\left(x-x_{m-1}\right)^{2}, & x \in\left[x_{m-1}, x_{m}\right], \\
\left(x-x_{m-1}\right)^{2}-3\left(x-x_{m}\right)^{2}, & x \in\left[x_{m}, x_{m+1}\right] \\
\left(x-x_{m-1}\right)^{2}-3\left(x-x_{m}\right)^{2}+3\left(x-x_{m+1}\right)^{2}, & x \in\left[x_{m+1}, x_{m+2}\right] \\
0, & \text { otherwise. }
\end{array}\right.
$$

The set of splines $\left\{Q_{-1}(x), Q_{0}(x), \ldots, Q_{N}(x)\right\}$ forms a basis for the functions defined over $[a, b]$. For this reason, an approximation solution $U_{N}(x, t)$ may be written in terms of the quadratic B-splines trial functions as:

$$
\mathrm{U}_{\mathrm{N}}(x, \mathrm{t})=\sum_{\mathrm{m}=-1}^{\mathrm{N}} \delta_{\mathrm{m}}(\mathrm{t}) \mathrm{Q}_{\mathrm{m}}(x)
$$

where $\delta_{\mathfrak{m}}(t)$ 's are time dependent parameters. Each quadratic B-spline involves three elements therefore every element of $\left[x_{m}, x_{m+1}\right]$ is coated with 
three quadratic B-splines. In this problem, the finite elements are described on the interval $\left[x_{\mathfrak{m}}, x_{\mathfrak{m}+1}\right]$ and the elements knots $x_{\mathfrak{m}}, x_{\mathfrak{m}+1}$. Using the nodal values $U_{m}$ and $U_{m}^{\prime}$ supplied in terms of the parameter $\delta_{m}(t)$

$$
\begin{aligned}
& \mathrm{U}_{\mathrm{N}}\left(\mathrm{x}_{\mathrm{m}}\right)=\mathrm{U}_{\mathrm{m}}=\delta_{\mathrm{m}-1}+\delta_{\mathrm{m}}, \\
& \mathrm{U}_{\mathrm{N}}^{\prime}\left(\mathrm{x}_{\mathrm{m}}\right)=\mathrm{U}_{\mathrm{m}}^{\prime}=2\left(-\delta_{\mathrm{m}-1}+\delta_{\mathrm{m}}\right) / \mathrm{h}
\end{aligned}
$$

the variation of $U_{N}(x, t)$ over the typical element $\left[x_{m}, x_{m+1}\right]$ is presented by

$$
\mathrm{u}_{N}(\xi, t)=\sum_{j=m-1}^{m+1} \delta_{j}(t) Q_{j}(\xi) .
$$

The Eq. (6) is the element equation for a typical element "e". Eq. (7) can be written as follows

$$
\underset{Q_{m+1}}{Q_{m-1}}=\frac{1}{h^{2}}\left\{\begin{array}{c}
(h-\xi)^{2}, \\
h^{2}+2 h \xi-2 \xi^{2}, \\
\xi^{2} .
\end{array}\right.
$$

Inserting equations Eqs. (9) into Eq. (6), we have

$$
\begin{aligned}
\sum_{j=m-1}^{m+1}\left[\int_{0}^{h} Q_{i} Q_{j} d \xi\right] \dot{\delta} & +\sum_{k=m-1}^{m+1} \sum_{j=m-1}^{m+1}\left[\int_{0}^{h} Q_{i} Q_{k}^{\prime} Q_{j} d \xi\right] \delta \\
& +v \sum_{j=m-1}^{m+1}\left[\int_{0}^{h} Q_{i}^{\prime} Q_{j}^{\prime} d \xi\right] \delta-\left.v \sum_{j=m-1}^{m+1}\left[Q_{i} Q_{j}^{\prime}\right] \delta\right|_{0} ^{h} \\
& =\int_{0}^{h} Q_{i} \tilde{f}(\xi, t) d \xi, \quad i=m-1, m, m+1
\end{aligned}
$$

where $\dot{\gamma}$ shows $\gamma^{\text {th }}$ order fractional derivative with respect to $t$. If we take

$$
\begin{aligned}
& A_{i j}^{e}=\int_{0}^{h} Q_{i} Q_{j} d \xi, B_{i k j}^{e}=\int_{0}^{h} Q_{i} Q_{k}^{\prime} Q_{j} d \xi, \\
& C_{i j}^{e}=\int_{0}^{h} Q_{i}^{\prime} Q_{j}^{\prime} d \xi, D_{i j}^{e}=\left.Q_{i} Q_{j}^{\prime}\right|_{0} ^{h}, E_{i}^{e}=\int_{0}^{h} Q_{i} \tilde{f}(\xi, t) d \xi
\end{aligned}
$$


Eq. (10) can be written in the matrix form

$$
A^{e} \dot{\delta}^{e}+B^{e} \delta^{e}+v C^{e} \delta^{e}-v D^{e} \delta^{e}=E^{e}
$$

where $\delta^{e}=\left(\delta_{m-1}, \delta_{\mathfrak{m}}, \delta_{m+1}\right)$. When the above integrations are calculated by using quadratic B-spline functions, we have

$$
\begin{gathered}
A_{i j}^{e}=\int_{0}^{h} Q_{i} Q_{j} d \xi=\frac{h}{30}\left[\begin{array}{ccc}
6 & 13 & 1 \\
13 & 54 & 13 \\
1 & 13 & 6
\end{array}\right], \\
B_{i k j}^{e}=\int_{0}^{h} Q_{i} Q_{k}^{\prime} Q_{j} d \xi=\frac{1}{30}\left[\begin{array}{ccc}
(-10,-19,-1) \delta^{e} & (8,12,0) \delta^{e} & (2,7,1) \delta^{e} \\
(-19,-54,-7) \delta^{e} & (12,0,-12) \delta^{e} & (7,54,19) \delta^{e} \\
(-1,-7,-2) \delta^{e} & (0,-12,-8) \delta^{e} & (1,19,10) \delta^{e}
\end{array}\right], \\
C_{i j}^{e}=\int_{0}^{h} Q_{i}^{\prime} Q_{j}^{\prime} d \xi=\frac{2}{3 h}\left[\begin{array}{ccc}
2 & -1 & -1 \\
-1 & 2 & -1 \\
-1 & -1 & 2
\end{array}\right], \\
D_{i j}^{e}=\left.Q_{i} Q_{j}^{\prime}\right|_{0} ^{h}=\frac{2}{h}\left[\begin{array}{ccc}
1 & -1 & 0 \\
1 & -2 & 1 \\
0 & -1 & 1
\end{array}\right] .
\end{gathered}
$$

where $i, j, k=m-1, m, m+1$. By writing the matrices $A, B, C, D$ and $E$ which are obtained by combining element matrixes in Eq. (11), we have the following matrix form equation:

$$
A \dot{\delta}+(B+v C-v D) \delta=E
$$

where $\delta=\left(\delta_{-1}, \delta_{0}, \delta_{1}, \ldots, \delta_{\mathrm{N}-1}, \delta_{\mathrm{N}}\right)$. If we write L1 formula

$$
\dot{\delta}_{m}=\frac{d^{\gamma} \delta}{d t^{\gamma}}=\frac{(\Delta t)^{-\gamma}}{\Gamma(2-\gamma)} \sum_{k=0}^{n-1}\left[(k+1)^{1-\gamma}-k^{1-\gamma}\right]\left[\delta_{m}^{n-k}-\delta_{m}^{n-k-1}\right],
$$

instead of $\dot{\delta}$ and Crank-Nicolson formula

$$
\delta_{\mathrm{m}}=\frac{1}{2}\left(\delta_{\mathrm{m}}^{\mathrm{n}}+\delta_{\mathrm{m}}^{\mathrm{n}+1}\right)
$$

instead of $\delta$, We have the recurrence correlation between sequential time levels about the unknown parameters $\delta_{m}^{n+1}(t)$

$$
\begin{aligned}
& {\left[A+(\Delta t)^{\gamma} \Gamma(2-\gamma)(B+v C-v D) / 2\right] \delta^{n+1}} \\
& =\left[A-(\Delta t)^{\gamma} \Gamma(2-\gamma)(B+v C-v D) / 2\right] \delta^{n} \\
& \quad-A \sum_{k=1}^{n}\left[(k+1)^{1-\gamma}-k^{1-\gamma}\right]\left[\delta^{n-k}-\delta^{n-k-1}\right]+(\Delta t)^{\gamma} \Gamma(2-\gamma) E
\end{aligned}
$$


$\delta=\left(\delta_{m-2}, \delta_{m-1}, \delta_{\mathfrak{m}}, \delta_{\mathfrak{m}+1}, \delta_{\mathrm{m}+2}\right)^{\mathrm{T}}$. The system (13) is composed of $\mathrm{N}+2$ linear equations that include unknown parameters $\mathrm{N}+2$. To achieve unique solution to these systems, we need two additional restrictions. These are obtained from the boundary conditions and can be used to eliminate $\delta_{-1}$ and $\delta_{N}$ from the systems. For this reason, we achieve a $\mathrm{N} \times \mathrm{N}$ solvable system of equations.

\section{Initial state}

The initial vector $\mathbf{d}^{0}=\left(\delta_{-1}, \delta_{0}, \delta_{1}, \ldots, \delta_{\mathrm{N}-2}, \delta_{\mathrm{N}-1}, \delta_{\mathrm{N}}\right)^{\mathrm{T}}$ is obtained by the initial and boundary conditions. Therefore, the approximation (8) can be rewritten for the initial condition as

$$
\mathrm{U}_{\mathrm{N}}(x, 0)=\sum_{\mathrm{m}=-1}^{\mathrm{N}} \delta_{\mathrm{m}}(0) \mathrm{Q}_{\mathrm{m}}(x)
$$

where the $\delta_{m}(0)$ 's are unknown parameters. We need the initial numerical approximation $\mathrm{U}_{\mathrm{N}}(\mathrm{x}, 0)$ provides the conditions:

$$
\begin{gathered}
\mathrm{U}_{\mathrm{N}}(x, 0)=\mathrm{U}\left(\mathrm{x}_{\mathrm{m}}, 0\right), \quad \mathrm{m}=0(1) \mathrm{N} \\
\mathrm{U}_{\mathrm{N}}^{\prime}\left(x_{0}, 0\right)=\mathrm{U}^{\prime}\left(x_{0}, 0\right) .
\end{gathered}
$$

So, using these conditions leads to a matrix system of the form

$$
W \mathbf{d}^{0}=\mathbf{b}
$$

where

$$
W=\left[\begin{array}{ccccccc}
\frac{-2}{h} & \frac{2}{h} & & & & & \\
1 & 1 & & & & & \\
& 1 & 1 & & & & \\
& & & \ddots & & & \\
& & & & 1 & 1 & \\
& & & & & 1 & 1
\end{array}\right]
$$

and

$$
\mathbf{b}=\left(\mathrm{u}^{\prime}\left(\mathrm{x}_{0}, 0\right), \mathrm{u}\left(\mathrm{x}_{0}, 0\right), \mathrm{u}\left(\mathrm{x}_{1}, 0\right), \ldots, \mathrm{U}\left(\mathrm{x}_{\mathrm{N}-2}, 0\right), \mathrm{U}\left(\mathrm{x}_{\mathrm{N}-1}, 0\right), \mathrm{u}\left(\mathrm{x}_{\mathrm{N}}, 0\right)\right)^{\mathrm{T}} .
$$

\section{$3 \quad$ Numerical examples and results}

In this section, we find the numerical solutions of problems which are obtained by quadratic B-spline Galerkin method. We calculate the accuracy of 
the method by the error norm $\mathrm{L}_{2}$

$$
L_{2}=\left\|\mathrm{u}^{\text {exact }}-\mathrm{u}_{\mathrm{N}}\right\|_{2} \simeq \sqrt{\frac{\mathrm{b}-\mathrm{a}}{\mathrm{N}} \sum_{j=0}^{\mathrm{N}}\left|\mathrm{u}_{j}^{\text {exact }}-\left(\mathrm{u}_{\mathrm{N}}\right)_{j}\right|^{2}}
$$

and the error norm $\mathrm{L}_{\infty}$

$$
L_{\infty}=\left\|\mathrm{u}^{\text {exact }}-\mathrm{U}_{\mathrm{N}}\right\|_{\infty} \simeq \max _{\mathrm{j}}\left|\mathrm{u}_{\mathrm{j}}^{\text {exact }}-\left(\mathrm{U}_{\mathrm{N}}\right)_{\mathrm{j}}\right| .
$$

Problem 1: Firstly, we consider the Eq. (1) with boundary conditions

$$
\mathrm{u}(0, \mathrm{t})=\mathrm{t}^{2}, \quad \mathrm{u}(1, \mathrm{t})=e \mathrm{t}^{2}, \quad \mathrm{t} \geq 0
$$

and the initial condition as

$$
\mathrm{U}(x, 0)=0, \quad 0 \leq x \leq 1 .
$$

The $f(x, t)$ is of the form

$$
f(x, t)=\frac{2 t^{2-\gamma} e^{x}}{\Gamma(3-\gamma)}+t^{4} e^{2 x}-v t^{2} e^{x} .
$$

The exact solution of the problem is given by

$$
u(x, t)=t^{2} e^{x} .
$$

The numerical solutions and the error norms for Problem 1 are given in Tables 1-3. If the results for $\gamma=0.50, \Delta t=0.00025, t=1, v=1$ and different number of partitions are examined in Table 1, one can see that when the number of partitions $\mathrm{N}$ are increased, the error norms $\mathrm{L}_{2}$ and $\mathrm{L}_{\infty}$ decrease significantly. The results which are obtained for $\gamma=0.50, \mathrm{~N}=80, \mathrm{t}=1$, $v=1$ and for different $\Delta t$ time steps are given in Table 2 . From this table it is clearly seen that when the $\Delta t$ time steps decrease, the error norms $\mathrm{L}_{2}$ and $\mathrm{L}_{\infty}$ decrease as it is expected. The results for different values of $\gamma, \Delta t=0.00025$, $\mathrm{N}=40, t=1, v=1$ are given with the error norms $\mathrm{L}_{2}$ and $\mathrm{L}_{\infty}$ in Table 3 . The error distributions obtained by quadratic B-spline Galerkin method for $\Delta t=0.00025, N=80, t=1, v=1$ and different values of $\gamma$ are given Fig. 1 . 
Table 1: Error norms and numerical solutions of Problem 1 for $\gamma=0.50$, $\Delta \mathrm{t}=0.00025, \mathrm{t}=1, v=1$.

\begin{tabular}{clllll}
\hline $\mathrm{x}$ & $\mathrm{N}=10$ & $\mathrm{~N}=20$ & $\mathrm{~N}=40$ & $\mathrm{~N}=80$ & Exact \\
\hline 0.0 & 1.000000 & 1.000000 & 1.000000 & 1.000000 & 1.000000 \\
0.1 & 1.105440 & 1.105287 & 1.105216 & 1.105197 & 1.105171 \\
0.2 & 1.222203 & 1.221644 & 1.221493 & 1.221455 & 1.221403 \\
0.3 & 1.351078 & 1.350217 & 1.349992 & 1.349935 & 1.349859 \\
0.4 & 1.493437 & 1.492287 & 1.491996 & 1.491922 & 1.491825 \\
0.5 & 1.650663 & 1.649270 & 1.648922 & 1.648838 & 1.648721 \\
0.6 & 1.824294 & 1.822727 & 1.822342 & 1.822247 & 1.822119 \\
0.7 & 2.016049 & 2.014378 & 2.013979 & 2.013882 & 2.013753 \\
0.8 & 2.227650 & 2.226118 & 2.225747 & 2.225661 & 2.225541 \\
0.9 & 2.461512 & 2.460020 & 2.459745 & 2.459680 & 2.459603 \\
1.0 & 2.718282 & 2.718282 & 2.718282 & 2.718282 & 2.718282 \\
\hline $\mathrm{L}_{2} \times 10^{3}$ & 1.632995 & 0.447720 & 0.161833 & 0.092624 & \\
$\mathrm{~L}_{\infty} \times 10^{3}$ & 2.296683 & 0.625018 & 0.227352 & 0.133125 & \\
\hline & & & & &
\end{tabular}

Table 2: Error norms and numerical solutions of Problem 1 for $\gamma=0.50, \mathrm{~N}=80$, $\mathrm{t}=1, v=1$.

\begin{tabular}{clllll}
\hline $\mathrm{x}$ & $\Delta \mathrm{t}=0.002$ & $\Delta \mathrm{t}=0.001$ & $\Delta \mathrm{t}=0.0005$ & $\Delta \mathrm{t}=0.00025$ & Exact \\
\hline 0.0 & 1.000000 & 1.000000 & 1.000000 & 1.000000 & 1.000000 \\
0.1 & 1.105356 & 1.105276 & 1.105236 & 1.105216 & 1.105171 \\
0.2 & 1.221768 & 1.221611 & 1.221533 & 1.221493 & 1.221403 \\
0.3 & 1.350395 & 1.350164 & 1.350049 & 1.349992 & 1.349859 \\
0.4 & 1.492516 & 1.492218 & 1.492070 & 1.491996 & 1.491825 \\
0.5 & 1.649543 & 1.649188 & 1.649011 & 1.648922 & 1.648721 \\
0.6 & 1.823031 & 1.822636 & 1.822440 & 1.822342 & 1.822119 \\
0.7 & 2.014687 & 2.014282 & 2.014080 & 2.013979 & 2.013753 \\
0.8 & 2.226387 & 2.226020 & 2.225837 & 2.225747 & 2.225541 \\
0.9 & 2.460180 & 2.459931 & 2.459807 & 2.459745 & 2.459603 \\
1.0 & 2.718282 & 2.718282 & 2.718282 & 2.718282 & 2.718282 \\
\hline $\mathrm{L}_{2} \times 10^{3}$ & 0.660788 & 0.375012 & 0.232768 & 0.092624 & \\
$\mathrm{~L}_{\infty} \times 10^{3}$ & 0.936619 & 0.530231 & 0.328303 & 0.133125 & \\
\hline
\end{tabular}


Table 3: Error norms and numerical solutions of Problem 1 for $\Delta t=0.00025, N=40$, $\mathrm{t}=1, v=1$.

\begin{tabular}{cccccl}
\hline$\chi$ & $\gamma=0.10$ & $\gamma=0.25$ & $\gamma=0.75$ & $\gamma=0.90$ & Exact \\
\hline 0.0 & 1.000000 & 1.000000 & 1.000000 & 1.000000 & 1.000000 \\
0.1 & 1.105218 & 1.105217 & 1.105216 & 1.105219 & 1.105171 \\
0.2 & 1.221497 & 1.221495 & 1.221493 & 1.221497 & 1.221403 \\
0.3 & 1.349997 & 1.349995 & 1.349990 & 1.349996 & 1.349859 \\
0.4 & 1.492001 & 1.492000 & 1.491993 & 1.492000 & 1.491825 \\
0.5 & 1.648930 & 1.648928 & 1.648920 & 1.648928 & 1.648721 \\
0.6 & 1.822351 & 1.822348 & 1.822339 & 1.822347 & 1.822119 \\
0.7 & 2.013987 & 2.013984 & 2.013977 & 2.013985 & 2.013753 \\
0.8 & 2.225751 & 2.225750 & 2.225744 & 2.225751 & 2.225541 \\
0.9 & 2.459747 & 2.459747 & 2.459744 & 2.459749 & 2.459603 \\
1.0 & 2.718282 & 2.718282 & 2.718282 & 2.718282 & 2.718282 \\
\hline $\mathrm{L}_{2} \times 10^{3}$ & 0.167077 & 0.165443 & 0.159924 & 0.166085 & \\
$\mathrm{~L}_{\infty} \times 10^{3}$ & 0.235837 & 0.232645 & 0.224523 & 0.232565 & \\
\hline
\end{tabular}

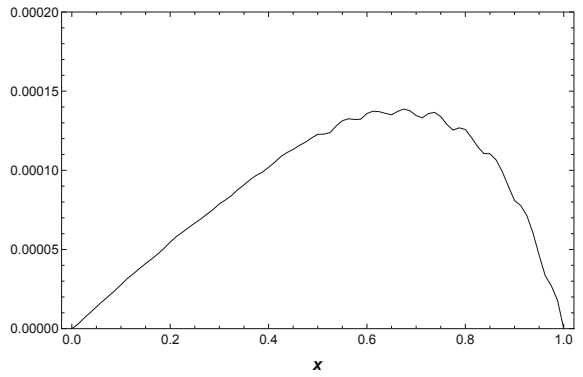

(a) $\gamma=0.25$

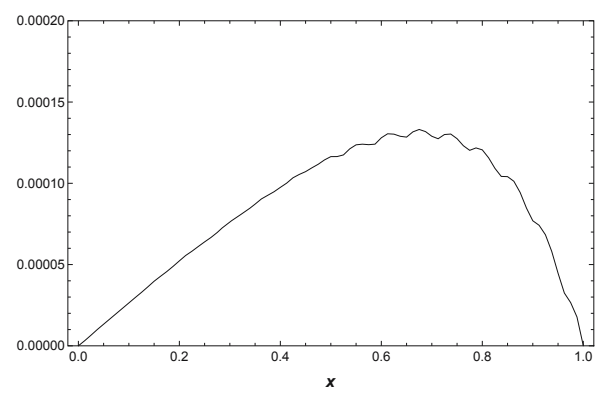

(b) $\gamma=0.50$

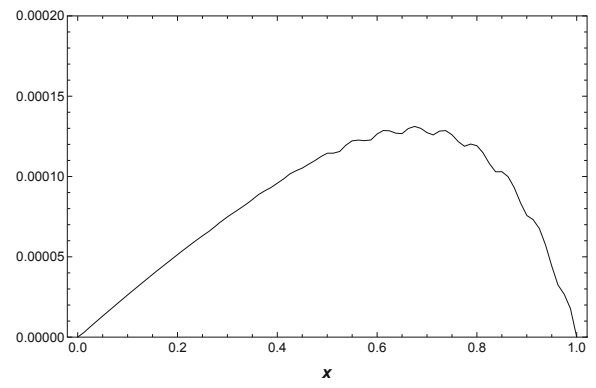

(c) $\gamma=0.75$

Figure 1: Error distributions of Problem 1 for $\Delta t=0.00025, N=80, t=1, v=1$. 
Problem 2: We secondly consider the Eq. (1), with boundary conditions

$$
\mathrm{u}(0, \mathrm{t})=\mathrm{t}^{2}, \quad \mathrm{u}(1, \mathrm{t})=-\mathrm{t}^{2}, \quad \mathrm{t} \geq 0
$$

and the initial condition as

$$
\mathrm{U}(x, 0)=0, \quad 0 \leq x \leq 1 .
$$

The term $f(x, t)$ is of the form

$$
f(x, t)=\frac{2 t^{2-\gamma} \cos (\pi x)}{\Gamma(3-\gamma)}-\pi t^{4} \cos (\pi x) \sin (\pi x)+v \pi^{2} t^{2} \cos (\pi x) .
$$

The exact solution of the problem is given by

$$
\mathrm{u}(x, \mathrm{t})=\mathrm{t}^{2} \cos (\pi x) .
$$

Numerical solutions and the error norms of Problem 2 which are achieved by the presented method for different values of division numbers, time steps, $v$ and $\gamma$ are given in Tables 4-7, respectively. When the tables are analyzed, it is easily seen that the numerical solutions converge to exact solution and the error norms $\mathrm{L}_{2}$ and $\mathrm{L}_{\infty}$ decrease considerably by increasing the number of division number, time step and decreasing the $v$. We give the error distributions of this method for different values of $\gamma, \Delta t=0.00025, N=80, t=1, v=1$ in Fig. 2 .

Table 4: Error norms and numerical solutions of Problem 2 for $\gamma=0.50, \Delta t=$ $0.00025, \mathrm{t}=1, v=1$.

\begin{tabular}{cccccc}
\hline $\mathrm{x}$ & $\mathrm{N}=10$ & $\mathrm{~N}=20$ & $\mathrm{~N}=40$ & $\mathrm{~N}=80$ & Exact \\
\hline 0.0 & 1.000000 & 1.000000 & 1.000000 & 1.000000 & 1.000000 \\
0.1 & 0.951278 & 0.950847 & 0.951005 & 0.951057 & 0.951057 \\
0.2 & 0.808287 & 0.808744 & 0.808954 & 0.809019 & 0.809017 \\
0.3 & 0.587257 & 0.587574 & 0.587738 & 0.587788 & 0.587785 \\
0.4 & 0.308724 & 0.308910 & 0.308993 & 0.309019 & 0.309017 \\
0.5 & 0.000000 & 0.000000 & 0.000000 & 0.000000 & 0.000000 \\
0.6 & -0.308724 & -0.308909 & -0.308996 & -0.309020 & -0.309017 \\
0.7 & -0.587257 & -0.587574 & -0.587741 & -0.587787 & -0.587785 \\
0.8 & -0.808286 & -0.808744 & -0.808957 & -0.809017 & -0.809017 \\
0.9 & -0.951277 & -0.950847 & -0.951008 & -0.951060 & -0.951057 \\
1.0 & -1.000000 & -1.000000 & -1.000000 & -1.000000 & -1.000000 \\
\hline $\mathrm{L}_{2} \times 10^{3}$ & 0.435334 & 0.183000 & 0.041977 & 0.001982 & \\
$\mathrm{~L}_{\infty} \times 10^{3}$ & 0.731099 & 0.273318 & 0.063233 & 0.004192 & \\
\hline
\end{tabular}


Table 5: Error norms and numerical solutions of Problem 2 for $\gamma=0.50, \mathrm{~N}=80$, $\mathrm{t}=1, v=1$.

\begin{tabular}{cccccc}
\hline $\mathrm{x}$ & $\Delta \mathrm{t}=0.002$ & $\Delta \mathrm{t}=0.001$ & $\Delta \mathrm{t}=0.0005$ & $\Delta \mathrm{t}=0.00025$ & Exact \\
\hline 0.0 & 1.000000 & 1.000000 & 1.000000 & 1.000000 & 1.000000 \\
0.1 & 0.951198 & 0.951117 & 0.951078 & 0.951057 & 0.951057 \\
0.2 & 0.809192 & 0.809093 & 0.809044 & 0.809019 & 0.809017 \\
0.3 & 0.587927 & 0.587848 & 0.587808 & 0.587788 & 0.587785 \\
0.4 & 0.309094 & 0.309051 & 0.309030 & 0.309019 & 0.309017 \\
0.5 & 0.000000 & 0.000000 & 0.000000 & 0.000000 & 0.000000 \\
0.6 & -0.309095 & -0.309052 & -0.309030 & -0.309020 & -0.309017 \\
0.7 & -0.587926 & -0.587847 & -0.587807 & -0.587787 & -0.587785 \\
0.8 & -0.809191 & -0.809092 & -0.809042 & -0.809017 & -0.809017 \\
0.9 & -0.951201 & -0.951120 & -0.951080 & -0.951060 & -0.951057 \\
1.0 & -1.000000 & -1.000000 & -1.000000 & -1.000000 & -1.000000 \\
\hline $\mathrm{L}_{2} \times 10^{3}$ & 0.124076 & 0.054112 & 0.019282 & 0.001982 & \\
$\mathrm{~L}_{\infty} \times 10^{3}$ & 0.175640 & 0.077491 & 0.028460 & 0.004192 & \\
\hline
\end{tabular}

Table 6: Error norms and numerical solutions of Problem 2 for $\gamma=0.50, \Delta t=0.0005$, $\mathrm{N}=80, \mathrm{t}=0.1$.

\begin{tabular}{ccccc}
\hline $\boldsymbol{x}$ & $\boldsymbol{v}=1$ & $\boldsymbol{v}=0.5$ & $\boldsymbol{v}=0.1$ & Exact \\
\hline 0.0 & 0.010000 & 0.010000 & 0.010000 & 0.010000 \\
0.1 & 0.009517 & 0.009517 & 0.009514 & 0.009511 \\
0.2 & 0.008099 & 0.008098 & 0.008095 & 0.008090 \\
0.3 & 0.005886 & 0.005885 & 0.005882 & 0.005878 \\
0.4 & 0.003095 & 0.003094 & 0.003092 & 0.003090 \\
0.5 & 0.000000 & 0.000000 & 0.000000 & 0.000000 \\
0.6 & -0.003095 & -0.003094 & -0.003092 & -0.003090 \\
0.7 & -0.005886 & -0.005885 & -0.005882 & -0.005878 \\
0.8 & -0.008099 & -0.008098 & -0.008095 & -0.008090 \\
0.9 & -0.009517 & -0.009517 & -0.009514 & -0.009511 \\
1.0 & -0.010000 & -0.010000 & -0.010000 & -0.010000 \\
\hline $\mathrm{L}_{2} \times 10^{3}$ & 0.006442 & 0.005834 & 0.003115 & \\
$\mathrm{~L}_{\infty} \times 10^{3}$ & 0.009009 & 0.008167 & 0.004425 & \\
\hline
\end{tabular}


Table 7: Error norms and numerical solutions of Problem 2 for $\Delta t=0.00025, N=80$, $\mathrm{t}=1, v=1$.

\begin{tabular}{cccccc}
\hline$x$ & $\gamma=0.10$ & $\gamma=0.25$ & $\gamma=0.75$ & $\gamma=0.90$ & Exact \\
\hline 0.0 & 1.000000 & 1.000000 & 1.000000 & 1.000000 & 1.000000 \\
0.1 & 0.951058 & 0.951058 & 0.951056 & 0.951057 & 0.951057 \\
0.2 & 0.809021 & 0.809020 & 0.809018 & 0.809019 & 0.809017 \\
0.3 & 0.587791 & 0.587789 & 0.587787 & 0.587788 & 0.587785 \\
0.4 & 0.309021 & 0.309020 & 0.309018 & 0.309019 & 0.309017 \\
0.5 & 0.000000 & 0.000000 & 0.000000 & 0.000000 & 0.000000 \\
0.6 & -0.309020 & -0.309020 & -0.309019 & -0.309020 & -0.309017 \\
0.7 & -0.587788 & -0.587788 & -0.587786 & -0.587787 & -0.587785 \\
0.8 & -0.809020 & -0.809018 & -0.809016 & -0.809017 & -0.809017 \\
0.9 & -0.951061 & -0.951060 & -0.951059 & -0.951060 & -0.951057 \\
1.0 & -1.000000 & -1.000000 & -1.000000 & -1.000000 & -1.000000 \\
\hline $\mathrm{L}_{2} \times 10^{3}$ & 0.003492 & 0.002733 & 0.001520 & 0.001886 & \\
$\mathrm{~L}_{\infty} \times 10^{3}$ & 0.006455 & 0.005257 & 0.003443 & 0.004065 & \\
\hline
\end{tabular}

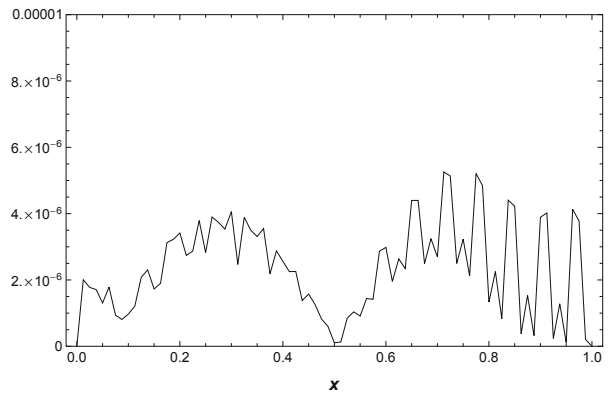

(a) $\gamma=0.25$

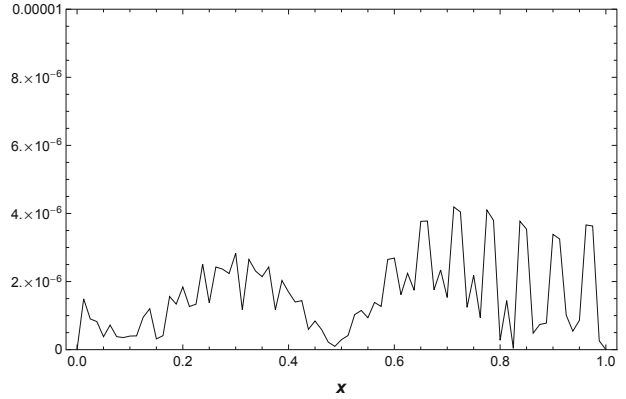

(b) $\gamma=0.50$

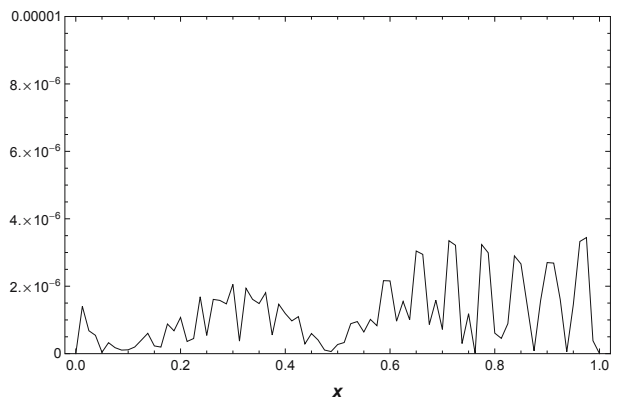

(c) $\gamma=0.75$

Figure 2: Error distributions of Problem 2 for $\Delta t=0.00025, N=80, t=1, v=1$. 
Problem 3: Finally, we consider the Eq. (1) with boundary conditions

$$
\mathrm{u}(0, \mathrm{t})=0, \quad \mathrm{u}(1, \mathrm{t})=0, \quad \mathrm{t} \geq 0
$$

and the initial conditions as

$$
\mathrm{u}(x, 0)=0, \quad 0 \leq x \leq 1 .
$$

The term $f(x, t)$ is of the form

$$
f(x, t)=\frac{2 t^{2-\gamma} \sin (2 \pi x)}{\Gamma(3-\gamma)}+2 \pi t^{4} \sin (2 \pi x) \cos (2 \pi x)+4 v \pi^{2} t^{2} \sin (2 \pi x) .
$$

The exact solution of the problem is given by

$$
\mathrm{u}(x, \mathrm{t})=\mathrm{t}^{2} \sin (2 \pi x) .
$$

Finally, error norms and numerical solutions for Problem 3 which calculated to test the accuracy of the solutions are given in Tables 8-11. The error norms and numerical solutions for different values of $\mathrm{N}, \gamma=0.50, \Delta t=0.00025, t=1$, $v=1$ are presented in Table 8 . From the table, it is understood that while the value of $\mathrm{N}$ is increasing, the error norms decrease. The results obtained for $\gamma=0.50, \mathrm{~N}=120, \mathrm{t}=1, v=1$, different time steps by this method are given in Table 9. From the table, it canbe seen that as $\Delta t$ time steps decrease, error norms decrease considerably. The tables show us that the numerical solutions are really close to the exact solutions. For $\Delta t=0.0005, N=120, t=1, v=1$ and different values of $v$ numerical solutions and error norms are given in Table 10. It shows us that while the value of $v$ is decreasing, the error norms decrease substantially. Again, for $\Delta t=0.0005, N=120, t=1, v=1$ and different values of $\gamma$, the result obtained by the presented method are given in Table 11. The error distributions achieved by the quadratic B-spline Galerkin method for $\Delta t=0.0005, N=120, t=1, v=1$ and different values of $\gamma$ are presented in Fig. 3. 
Table 8: Error norms and numerical solutions of Problem 3 for $\gamma=0.50, \Delta t=$ $0.00025, t=1, v=1$.

\begin{tabular}{crrrrr}
\hline $\mathrm{x}$ & $\mathrm{N}=40$ & $\mathrm{~N}=50$ & $\mathrm{~N}=80$ & $\mathrm{~N}=100$ & \multicolumn{1}{c}{ Exact } \\
\hline 0.0 & 0.000000 & 0.000000 & 0.000000 & 0.000000 & 0.000000 \\
0.1 & 0.585106 & 0.586153 & 0.587257 & 0.587505 & 0.587785 \\
0.2 & 0.947079 & 0.948617 & 0.950262 & 0.950638 & 0.951057 \\
0.3 & 0.947320 & 0.948761 & 0.950310 & 0.950666 & 0.951057 \\
0.4 & 0.585586 & 0.586434 & 0.587348 & 0.587562 & 0.587785 \\
0.5 & 0.000001 & -0.000002 & 0.000000 & 0.000007 & 0.000000 \\
0.6 & -0.585584 & -0.586437 & -0.587346 & -0.587548 & -0.587785 \\
0.7 & -0.947318 & -0.948767 & -0.950310 & -0.950661 & -0.951057 \\
0.8 & -0.947078 & -0.948621 & -0.950260 & -0.950631 & -0.951057 \\
0.9 & -0.585106 & -0.586155 & -0.587257 & -0.587503 & -0.587785 \\
1.0 & 0.000000 & 0.000000 & 0.000000 & 0.000000 & 0.000000 \\
\hline $\mathrm{L}_{2} \times 10^{3}$ & 2.899412 & 1.774196 & 0.577143 & 0.305058 & \\
$\mathrm{~L}_{\infty} \times 10^{3}$ & 4.063808 & 2.495647 & 0.813220 & 0.430014 & \\
\hline
\end{tabular}

Table 9: Error norms and numerical solutions of Problem 3 for $\gamma=0.50, \mathrm{~N}=120$, $\mathrm{t}=1, v=1$.

\begin{tabular}{cccccc}
\hline $\mathrm{x}$ & $\Delta \mathrm{t}=0.0025$ & $\Delta \mathrm{t}=0.002$ & $\Delta \mathrm{t}=0.001$ & $\Delta \mathrm{t}=0.0005$ & Exact \\
\hline 0.0 & 0.000000 & 0.000000 & 0.000000 & 0.000000 & 0.000000 \\
0.1 & 0.588970 & 0.588675 & 0.588083 & 0.587788 & 0.587785 \\
0.2 & 0.952952 & 0.952484 & 0.951545 & 0.951076 & 0.951057 \\
0.3 & 0.952914 & 0.952458 & 0.951544 & 0.951086 & 0.951057 \\
0.4 & 0.588914 & 0.588635 & 0.588087 & 0.587810 & 0.587785 \\
0.5 & 0.000005 & 0.000005 & 0.000005 & 0.000004 & 0.000000 \\
0.6 & -0.588905 & -0.588630 & -0.588077 & -0.587801 & -0.587785 \\
0.7 & -0.952912 & -0.952456 & -0.951540 & -0.951084 & -0.951057 \\
0.8 & -0.952949 & -0.952479 & -0.951540 & -0.951070 & -0.951057 \\
0.9 & -0.588968 & -0.588672 & -0.588080 & -0.587784 & -0.587785 \\
1.0 & 0.000000 & 0.000000 & 0.000000 & 0.000000 & 0.000000 \\
\hline $\mathrm{L}_{2} \times 10^{3}$ & 1.392372 & 1.048597 & 0.359489 & 0.017828 & \\
$\mathrm{~L}_{\infty} \times 10^{3}$ & 1.974356 & 1.487805 & 0.512105 & 0.032162 & \\
\hline
\end{tabular}


Table 10: Error norms and numerical solutions of Problem 3 for $\gamma=0.50, \Delta t=$ $0.0005, \mathrm{~N}=120, \mathrm{t}=0.1$.

\begin{tabular}{ccccccc}
\hline$x$ & $v=1$ & $v=0.5$ & $v=0.1$ & $v=0.01$ & $v=0.005$ & Exact \\
\hline 0.0 & 0.000000 & 0.000000 & 0.000000 & 0.000000 & 0.000000 & 0.000000 \\
0.1 & 0.005902 & 0.005900 & 0.005890 & 0.005879 & 0.005878 & 0.005878 \\
0.2 & 0.009550 & 0.009546 & 0.009531 & 0.009512 & 0.009510 & 0.009511 \\
0.3 & 0.009550 & 0.009546 & 0.009531 & 0.009512 & 0.009510 & 0.009511 \\
0.4 & 0.005902 & 0.005900 & 0.005890 & 0.005878 & 0.005877 & 0.005878 \\
0.5 & 0.000000 & 0.000000 & 0.000000 & 0.000000 & 0.000000 & 0.000000 \\
0.6 & -0.005902 & -0.005900 & -0.005890 & -0.005878 & -0.005876 & -0.005878 \\
0.7 & -0.009550 & -0.009546 & -0.009531 & -0.009512 & -0.009510 & -0.009511 \\
0.8 & -0.009550 & -0.009546 & -0.009531 & -0.009512 & -0.009510 & -0.009511 \\
0.9 & -0.005902 & -0.005900 & -0.005890 & -0.005879 & -0.005878 & -0.005878 \\
1.0 & 0.000000 & 0.000000 & 0.000000 & 0.000000 & 0.000000 & 0.000000 \\
\hline $\mathrm{L}_{2} \times 10^{3}$ & 0.029174 & 0.026666 & 0.015017 & 0.001045 & 0.000758 & \\
$\mathrm{~L}_{\infty} \times 10^{3}$ & 0.041294 & 0.037739 & 0.021269 & 0.002001 & 0.002341 & \\
\hline
\end{tabular}

Table 11: Error norms and numerical solutions of Problem 3 for $\Delta t=0.0005, N=$ $120, t=1, v=1$.

\begin{tabular}{crrrrr}
\hline$\chi$ & $\gamma=0.10$ & $\gamma=0.25$ & $\gamma=0.75$ & $\gamma=0.90$ & \multicolumn{1}{c}{ Exact } \\
\hline 0.0 & 0.000000 & 0.000000 & 0.000000 & 0.000000 & 0.000000 \\
0.1 & 0.586505 & 0.587787 & 0.587788 & 0.587791 & 0.587785 \\
0.2 & 0.950362 & 0.951076 & 0.951078 & 0.951082 & 0.951057 \\
0.3 & 0.950933 & 0.951088 & 0.951088 & 0.951092 & 0.951057 \\
0.4 & 0.587791 & 0.587813 & 0.587811 & 0.587814 & 0.587785 \\
0.5 & 0.000000 & 0.000007 & 0.000005 & 0.000004 & 0.000000 \\
0.6 & -0.587833 & -0.587798 & -0.587802 & -0.587804 & -0.587785 \\
0.7 & -0.951333 & -0.951080 & -0.951085 & -0.951089 & -0.951057 \\
0.8 & -0.952217 & -0.951068 & -0.951072 & -0.951076 & -0.951057 \\
0.9 & -0.589827 & -0.587784 & -0.587785 & -0.587788 & -0.587785 \\
1.0 & 0.000000 & 0.000000 & 0.000000 & 0.000000 & 0.000000 \\
\hline $\mathrm{L}_{2} \times 10^{3}$ & 0.879696 & 0.017780 & 0.018641 & 0.021398 & \\
$\mathrm{~L}_{\infty} \times 10^{3}$ & 2.051516 & 0.034072 & 0.033291 & 0.037357 & \\
\hline
\end{tabular}




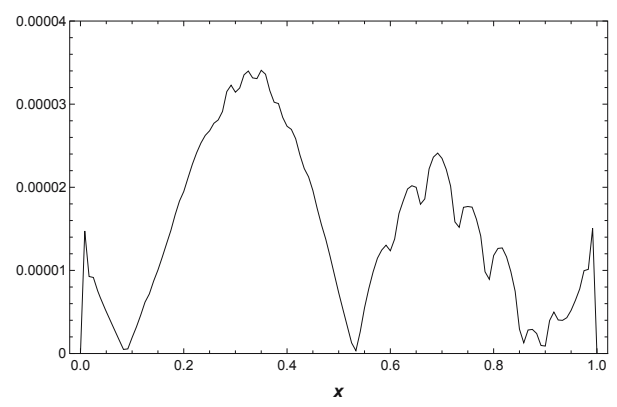

(a) $\gamma=0.25$

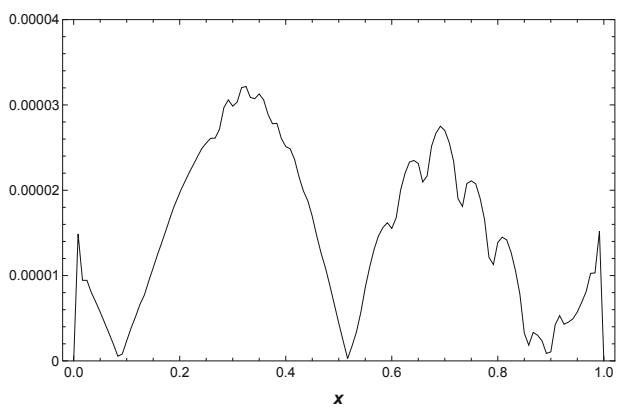

(b) $\gamma=0.50$

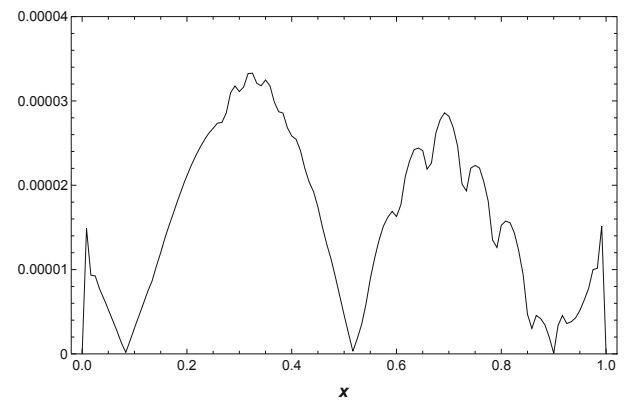

(c) $\gamma=0.75$

Figure 3: Error distributions of Problem 3 for $\Delta t=0.0005, N=120, t=1, v=1$.

\section{Conclusion}

In this paper, quadratic B-spline Galerkin method has been applied to acquire the numerical solutions of three problems for the time fractional Burgers equation. The time fractional derivative operator is made allowance for the Caputo fractional derivative in these problems. It can be easily viewed from the numerical solutions and error norms in tables obtained that this is an extremely good method to achieve numerical solutions of time fractional partial differential equations arising in physics and engineering. 


\section{References}

[1] L. Debnath, Partial Differential Equations for Scientists and Engineers, Birkhäuser, Boston, 1997.

[2] S. Kutluay, A. Esen, I. Dag, Numerical solutions of the Burgers' equation by the least squares quadratic B-spline finite element method, J. Comp. Appl. Math., 167 (2004) 21-33.

[3] L. R. T. Gardner, G. A. Gardner, A. Dogan, A Petrov-Galerkin finite element scheme for Burgers equation, Arab. J. Sci. Eng., 22 (1997) 99109.

[4] A. H. A. Ali, L. R. T. Gardner, G. A. Gardner, A Collocation method for Burgers equation using cubic splines, Comp. Meth. Appl. Mech. Eng., (1992) 325-337.

[5] K. R. Raslan, A collocation solution for Burgers equation using quadratic B-spline finite elements, Intern. J. Computer Math., 80 (2003) 931-938.

[6] I. Dag, D. Irk, B. Saka, A numerical solution of Burgers equation using cubic B-splines, Appl. Math. Comput., 163 (2005) 199-211.

[7] M. A. Ramadan, T. S. El-Danaf, F. E. I. Abd Alaal, Numerical solution of Burgers equation using septic B-splines, Chaos, Solitons and Fractals, 26 (2005) 795-804.

[8] N. Sugimoto, Burgers equation with a fractional derivative; hereditary effects on nonlinear acoustic waves, J. Fluid Mech., 225 (1991) 631-653.

[9] P. Miskinis, Some Properties of Fractional Burgers Equation, Mathematical Modelling and Analysis, 7 (2002) 151-158.

[10] S. Momani, Non-perturbative analytical solutions of the space- and timefractional Burgers equations, Chaos, Solitons and Fractals, 28 (2006) 930937.

[11] M. Inc, The approximate and exact solutions of the space- and timefractional Burgers equations with initial conditions by variational iteration method, J. Math. Anal. Appl. 345 (2008) 476-484.

[12] C. Li, Y. Wang, Numerical algorithm based on Adomian decomposition for fractional differential equations, Computers and Mathematics with Applications, 57 (2009) 1672-1681. 
[13] T. S. El-Danaf, A. R. Hadhoud, Parametric spline functions for the solution of the one time fractional Burgers equation, Applied Mathematical Modelling, 36 (2012) 4557-4564.

[14] M. Younis, A. Zafar, Exact Solution to Nonlinear Differential Equations of Fractional Order via ( $\left.\mathrm{G}^{\prime} / \mathrm{G}\right)$-Expansion Method, Applied Mathematics, 5 (2014) 1-6.

[15] D. L. Logan, A First Course in the Finite Element Method (Fourth Edition), Thomson, 2007.

[16] K. B. Oldham, J. Spanier, The Fractional Calculus, Academic, New York, 1974.

[17] A. Esen, Y. Ucar, N. Yagmurlu, O Tasbozan, A Galerkin Finite Element Method to Solve Fractional Diffusion and Fractional Diffusion-Wave Equations, Mathematical Modelling and Analysis, 18 (2013) 260-273.

[18] O. Tasbozan, A. Esen, N. M. Yagmurlu, Y. Ucar, A Numerical Solution to Fractional Diffusion Equation for Force-Free Case, Abstr. Appl. Anal. (2013), http://dx.doi.org/10.1155/2013/187383, Article ID 187383, 6 pp.

[19] F. Liu, P. Zhuang, I. Turner, K. Burrage, V. Anh, A new fractional finite volume method for solving the fractional diffusion equation, Applied Mathematical Modelling, 38 (2014) 3871-3878.

[20] H. Zhang, F. Liu, P. Zhuang, I. Turner, V. Anh, Numerical analysis of a new space-time variable fractional order advection-dispersion equation, Applied Mathematics and Computation, 242 (2014) 541-550.

[21] A. Pedas, E. Tamme, Numerical solution of nonlinear fractional differential equations by spline collocation method, Journal of Computational and Applied Mathematics, 255 (2014) 216-230.

[22] S. Butera, M. D. Paola, Fractional differential equations solved by using Mellin transform, Commun. Nonlinear. Sci. Numer. Simulat., 19 (2014) $2220-2227$.

[23] J. Zhao, Jingyu Xiao, N. J. Ford, Collocation methods for fractional integro-differential equations with weakly singular kernels, Numer. Algor., 65 (2014) 723-743. 
[24] A. B. Orovio, D. Kay, K. Burrage, Fourier spectral methods for fractional in space reaction-diffusion equations, BIT Numer. Math., 54 (2014) 937954.

[25] S. Tatar, S. Ulusoye, An inverse source problem for a one-dimensional space-time fractional diffusion equation, Applicable Analysis, (2014).

[26] F. F. Dou, Y. C. Hon, Numerical computation for backward timefractional diffusion equation, Engineering Analysis with Boundary Elements, 40 (2014) 138-146.

[27] B. Cockburn, K. Mustapha, A hybridizable discontinuous Galerkin method for fractional diffusion problems, Numer. Math., (2014).

[28] Y. Zhanga, Z. Sunb, H. Liaoc, Finite difference methods for the time fractional diffusion equation on non-uniform meshes, Journal of Computational Physics, 265 (2014) 195-210.

[29] A. Esen, Y. Ucar, M. Yagmurlu, O. Tasbozan, Solving Fractional Diffusion and Fractional Diffusion-Wave Equations by Petrov-Galerkin Finite Element Method, TWMS J. App. Eng. Math., 4 (2014) 155-168.

[30] A. Esen, O. Tasbozan, An approach to Time Fractional Gas Dynamics Equation: Quadratic B-Spline Galerkin Method, Applied Mathematics and Computation, 261 (1) (2015) 330-336.

[31] A. Esen, O. Tasbozan, Y. Ucar, N. M. Yagmurlu, A B-spline collocation method for solving fractional diffusion and fractional diffusion-wave equations, Tbilisi Mathematical Journal, 8 (2) (2015) 181-193.

[32] I. Podlubny, Fractional Differential Equations, Academic Press, San Diego, 1999.

[33] P. M. Prenter, Splines and Variasyonel Methods, New York, John Wiley, 1975. 